\title{
Lasers and dento-facial orthopedics: an interview with Samir Nammour
}

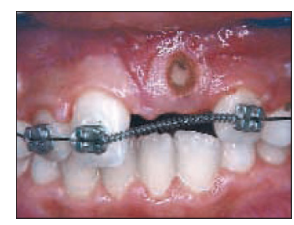

\section{Philippe AMAT, Jacques BERREBI, Franck HERVÉ}

Samir Nammour is a professor at the Dental School of the University of Liege. A specialist in the use of lasers in dentistry, he served as president of the World Federation for Laser Dentistry (WFLD) until 1908 and is currently the director of the European Masters Degree in Oral Laser Applications program.

Philippe Amat, Jacques BerReBI, Franck Hervé. Professor Sam Nammour, you are the director of the European Masters Degree in Oral Laser Applications program. Because of your contributions to it, all its participants have awarded you with a bronze star prize. This honor presented within the framework of the European Life-Long Education and Training program, reflects the recognition throughout the continent of $3^{\text {rd }}$ cycle diplomas for dentists. Can you tell us about the modalities and objectives of this program?

Samir Nammour. The three universities of Nice, in France, of Aix-la-Chapelle in Germany, and Liege in Belgium, have, independently, been offering courses in the use of lasers in dentistry since the year
2000. Then one day we began to wonder why we should all be working, isolated, in our private academic fief-doms? Why couldn't we pool our resources and present a single, more comprehensive, unified course? We presented this border breaching concept to the European Community, asking that the three universities be authorized to offer a common diploma to be called the European Masters Degree in Oral Laser Applications, (EMDOLA). After two years of joint teaching experience, we were delighted to learn that the European Community was ready to grant final acceptance of our project. We hope to continue offering the highest quality instruction that would unite the best potentials developed in each of the three participating institutions. 
P.A., J.B., F.H. Do you plan to introduce the EMDOLA project in other European countries?

S.N. That's a good question. In September 2008, three new universities, Parma and Rome in Italy, and Barcelona in Spain, joined our EMDOLA group, which now embraces six schools. We have had to reject the application of two additional universities but we hope they will have improved their standards sufficiently for us to accept them next year.

P.A., J.B., F.H. What are the possibilities of inter-university student exchange studies within your EMDOLA group?

S.N. Our goal is to make such exchanges readily available. We have organized instruction in modules presented at staggered dates so that students will be able to attend a widely diverse range of courses in different universities.

In addition we have coordinated our efforts to that teachers will be giving comparable courses in all the participating schools, with courses given alternatively at each university.

P.A., J.B., F.H. Before we go into greater detail about the indications for the use of each of them, can you outline for us the characteristics of the different types of lasers, $\mathrm{CO}_{2}$, Erbium Yag, and others that are utilized in dentistry?

S.N. The therapeutic application of the Carbon Dioxide laser, $\mathrm{CO}_{2}$, laser is derived from its capability of instantaneously releasing an intense burst of heat that can fuse hard dental tissues. Its capability of in- stantly evaporating soft tissue and executing extremely precise, totally bloodless, cuts and excisions makes it a useful surgical tool as well.

As for the laser Erbium Yag, it is primarily characterized by its power of sending a light ray that, in contact with hard tissue, provokes a micro-explosion, which, when repeated, allows dentists to progressively remove hard tissue.

P.A., J.B., F.H. What are the principal applications of lasers in dentistry?

S.N. The $\mathrm{CO}_{2}$. laser has become the instrument of choice in oral surgery. Its indications, advantages, and limits have been clearly understood for many years.

The Erbium-Yag (Er: Yag) laser is primarily employed in conservative dentistry thanks to its efficacy in removing hard tissue. Although it is less efficient and slower in cutting through soft tissue, it can still be employed surgically, espe cially in reshaping gingival tissues.

The fiber lasers, like the NéodymeYag (Nd: Yag), and the Néodyme-Yap (Nd: Yap), the only laser made in France, as well as the diodes of varying lengths, have much more limited applications. They are employed in endodontics because of their capability of providing light in areas of difficult access through an uncovered tip. Acting in the interior of root chambers they warm disinfecting liquids such as sodium hypochlorite, which enhances their effect, while at the same time removing infected tissue. This action is accompanied by vibrations that participate in eliminat- 
ing the smear layer as well as in diffusing the disinfections products further into the canal complex of tubules and secondary canals.

Periodontists can employ fiber lasers to cleanse supra or infra-osseous periodontal pockets. In the supra-osseous pockets, the beneficial action of the lasers cans be seen on three levels.

- First, they restore health to the gingiva by reducing acute inflammation. This effect, which is immediate and quite spectacular, becomes evident as the gingiva changes in color;

- Second, lasers provide a decontamination action, whose effectiveness depends on the wave length employed;

- And, finally, lasers set off a biostimulation effect that promotes cellular regeneration and improvement in gingival health.

But it should be emphasized that use of lasers in no way relieves clinicians from performing tried and tested basic treatment like prophylactic removal of tartar.

P.A., J.B., F.H. You have written several articles ${ }^{8},{ }^{9}$ about using the Argon laser to improve dental enamel's capacity to retain fluoride coating. Can you outline for us the clinical applications of this technique as well as the current status of your research?

S.N. Dental caries, quite properly considered to be a scourge, has a high worldwide prevalence. We hope to be able to contribute to the prevention of this malady by a fluoride enrichment technique, whose modest cost, we anticipate, will make it readily available to great numbers of people, no matter how limited resources or how distressed local conditions may be.

After application of a fluoride gel on teeth, our technique consists of irradiating the dental surface with an Argon laser. After 30 seconds of exposure to this beam, a sufficiently large amount of fluoride will be incorporated immediately and permanently into the enamel. We are at present proposing this protocol to our patients.

P.A., J.B., F.H. Can you tell us whether you are now at the preliminary trial stage or have you already begun clinical applications of your method?

S.N. After having obtained excellent results with numerous clinical studies, I think I can safely say we have now reached the stage of applying our tech nique clinically.

P.A., J.B., F.H. You are one of the co-authors of a recent clinical study published in Photomedicine and Laser Surgery ${ }^{2}$ that was devoted to the applications of the laser Nd:YAG and $810 \mathrm{~nm}$ and $980 \mathrm{~nm}$ diodes in soft tissue orthodontie surgery. What are the advantages of this new operative technique compared to conventional ones? Have you observed a decrease in post-operative pain or in the time required for healing?

S.N. The use of lasers for soft tissue surgery provides no benefits with respect to post-operative pain or length of the healing process. In fact, the sutures that conclude conventional surgery assure a more rapid first intention healing. But the advantage of the laser technique lies precisely there: the wound requires no sutures, but the second intention healing time is also longer. 
The article that your question calls up illustrates the two principal advantages of laser fibers; the operative procedure is completely bloodless because the laser seals off capillaries and it can be performed in areas for which access would otherwise be extremely difficult or impossible. A special case where elimination of bleeding is extremely helpful is exposure of impacted teeth, which, because of the dry field, are immediately available for successful bonding of attachments, allowing orthodontic movement to begin without delay.

P.A., J.B., F.H. You are saying, then, that fiber lasers are the instrument of choice, particularly in orthodontics, because they can be used in areas that would not be accessible to other lasers, like $\mathrm{CO}_{2}$ ?

S.N. Exactly. The rays of light that emerge from a $\mathrm{CO}_{2}$ cannot be carried along by a fiber. For those areas where access is difficult, it is, therefore, sometimes necessary to use another wave length (lasers Nd:YAG, Nd:YAP or Diodes), even if they are otherwise less appropriate for that procedure. They are less efficient on soft tissue because their water content does not allow them to be readily absorbable.

P.A., J.B., F.H. Can you explain for our readers why a laser rather than a conventional surgical procedure should be selected for an operation on a deeply inserted frenum?

S.N. Lasers give dentists a fine cutting edge that allows them to pass safely between the teeth to vaporize the ensemble of fibrous soft tissue right up to their insertion into bone. The surgical site is completely cleared of blood and the sterilizing power of the laser greatly reduces the risk of post-operative infection (fig. 1 a to $\mathrm{c}$ and fig. 2).

Another advantage is that pain is less than in the traditional way of vigorously curetting the fibrous insertions in the inter-maxillary suture. This curettage, which, of necessity, must be pursued well into the bone causes intense post-operative pain that must be relieved with analgesics over a period of several days. But patients require pain medication when lasers are used only on the day of the surgery and, perhaps for a half-day afterwards.

P.A., J.B., F.H. Do these considerations apply to the resection of a lingual frenum?

S.N. Absolutely. For this type of procedure the $\mathrm{CO}_{2}$ laser provides practitioners with great cutting precision, excellent hemostasis, and a significant reduction in post-operative pain (fig. 3 a to $C$, and 4 a to e).

P.A., J.B., F.H. What benefits do you think lasers bring to the surgical-orthodontic uncovering of an impacted tooth, a canine, for example?

S.N. In these cases, their indication is superb and truly original. If, for example laser execution of a frenectomy presents undeniable advantages, frenectomies can be accomplished perfectly adequately by conventional surgery.

But lasers uncovering impacted teeth (fig. 5 a to f) work so much better than conventional surgery that it would be unconscionable not to allow our patients to benefit from them because, as I have said, they allow orthodontic bonding and traction to begin immediately thanks to the hemostasis of the operative field. 


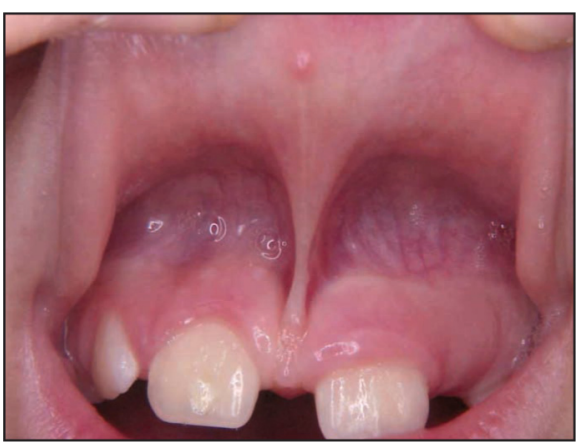

Figure 1 a

Maxillary median labial frenum with a low insertion (documents Fornaini, et $a l^{2}{ }^{2}$.

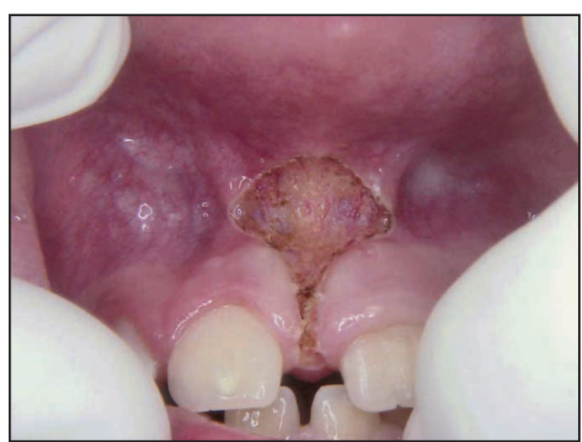

Figure $1 \mathrm{~b}$

Use of a Diode to vaporize all fibrous tissue right up to its insertion in bone. Note the perfect hemostasis obtained in their surgical site.

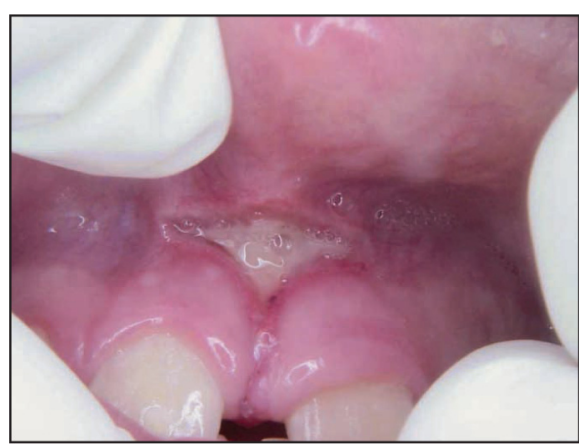

Figure $1 \mathrm{c}$

View of the site 7 days after the surgical procedure. The presence of a fibrous pellicle indicates that healing is proceeding satisfactorily.

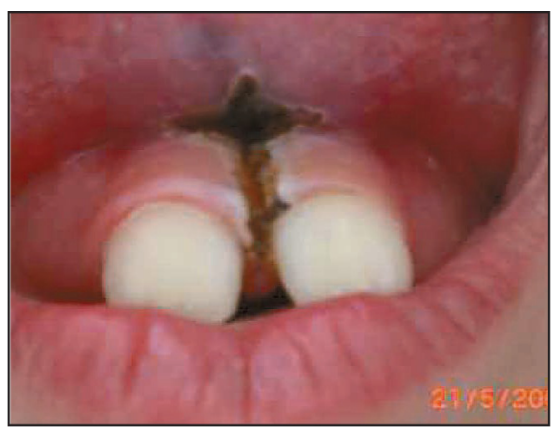

Figure 2

For another patient the surgeon has used a long wave $\mathrm{CO}_{2}$ laser in order to make a very fine incision between the teeth without injuring them (from the records of J. Berrebi).

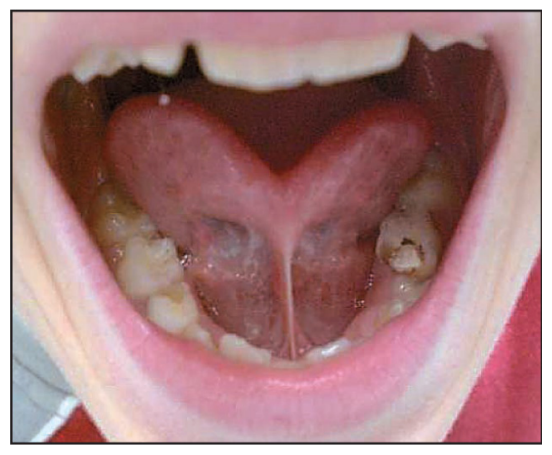

Figure 3 a

Extremely tight, or ankylosed lingual frenum (from the records of $\mathrm{J}$. Berrebi).

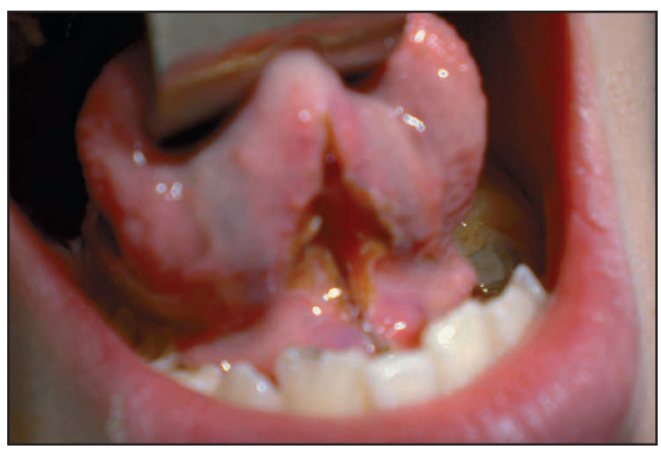

Figure $3 b$

Concentrated pulsations from a $\mathrm{CO}_{2}$ laser have freed the frenum from its two attachments, to the tongue and to the lingual incisal mucosa. In this view taken immediately after the laser procedure, the perfect hemostasis in this highly vascularized region can clearly be seen.

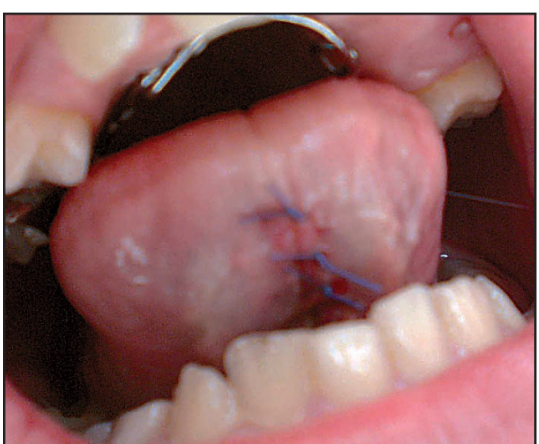

Figure $3 c$

The tongue demonstrates the great extent of its newly achieved mobility. 


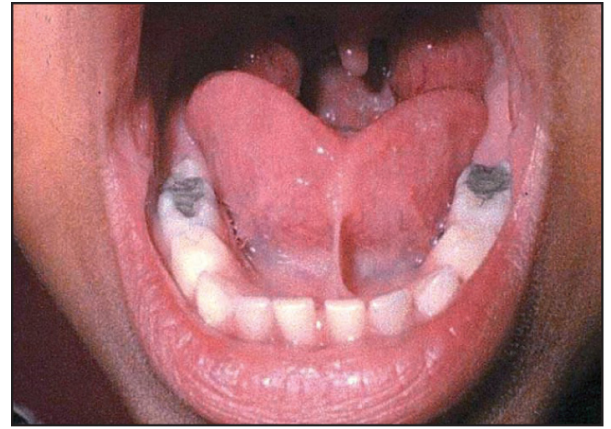

Figure 4 a

A Short lingual frenum that deforms the point of the tongue giving it the contour of a heart shaped playing card (from the records if $S$. Nammour).

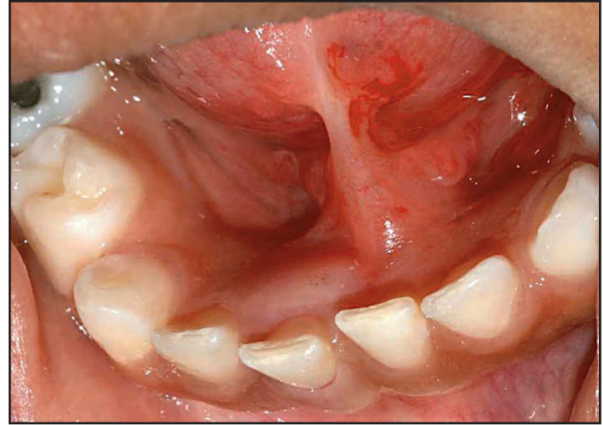

Figure $4 b$

A Close-up view of that same thick, short, and fibrous lingual frenum.

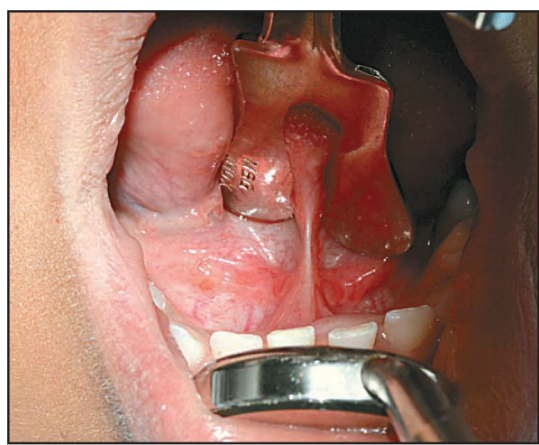

Figure $4 \mathrm{c}$

A view of the operating site after its isolation.

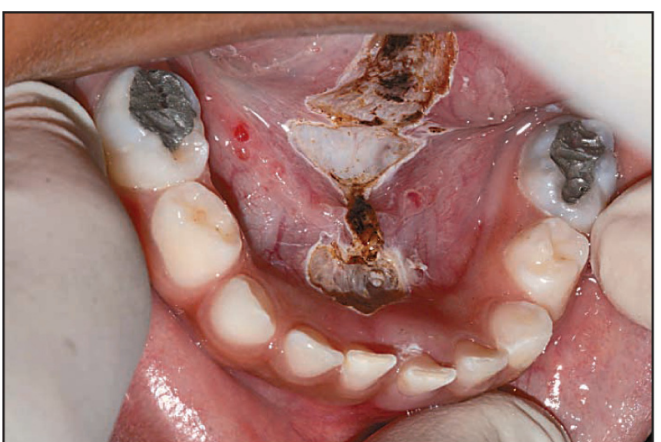

Figure $4 d$

A view of the site taken immediately after the operation. The slight carbonization of the site, always superficial with a $\mathrm{CO}_{2}$ laser, is normal for this stage.

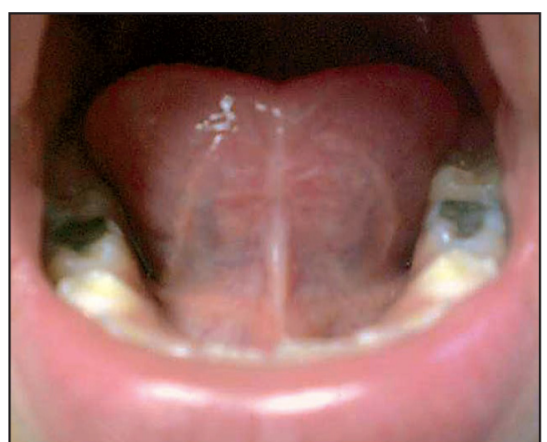

Figure $4 e$ The result after 60 days.
P.A., J.B., F.H. Another indication for the use of lasers in orthodontics is in the treatment of gingival hyperplasia, which we see principally in patients who are unable to effectively control build up of dental plaque in their mouths. Is the presence of metal brackets on patients' teeth a contra-indication for removing excessive soft tissue?

S.N. Dentists can, perhaps, obviate this obstacle by using lasers, like $\mathrm{CO}_{2}$, whose wave lengths metal does not absorb but reflects as though the metal were a mirror. This type of wave length, located in the infra-red area, does not heat metal at all, meaning they pose no threat to the integrity of the bonds of attachments.

Thanks to the $\mathrm{CO}_{2}$ laser dentists can recontour gingival margins with precision and with no risk of a postoperative gingival recession occurring, thus allowing patients to return to the 


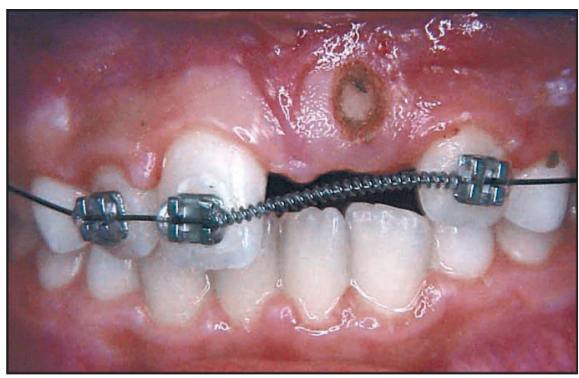

Figure 5 a

Surgical uncovering with a laser of an impacted maxillary central incisor (from the records of Fornaini, et $a l^{2}$ ).

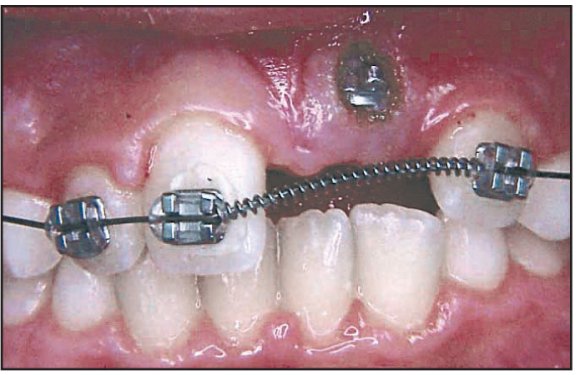

Figure $5 b$

In the same visit, thanks to the laser imposed hemostasis of the operating field, an attachment was effectively bonded to the exposed labial surface of the tooth.

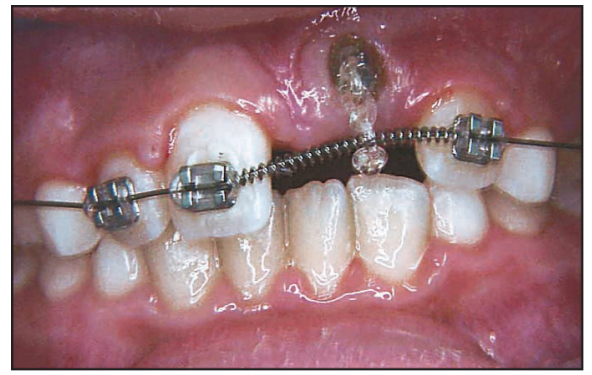

Figure $5 c$

Orthodontic movement of the impacted tooth was begun by tying it to the arch wire with an elastic ligature.

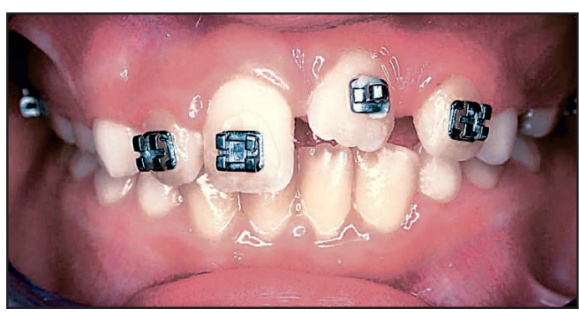

Figure $5 d$

Termination of initial stage of orthodontic movement of the impacted tooth.

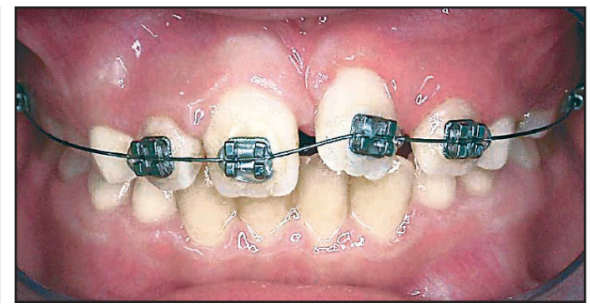

Figure 5 e

The hook type attachment was replaced with a bracket to allow for final positioning of the impacted tooth.

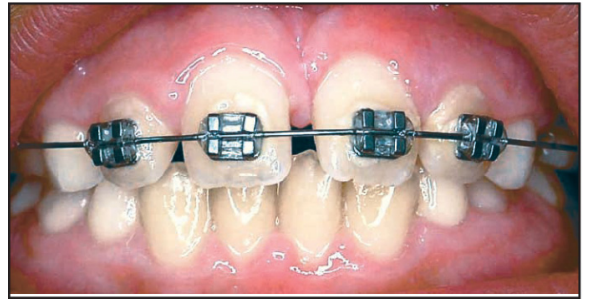

Figure $5 f$

After movement with a light wire the impacted tooth has assumed its position in the arch. desired impeccable oral hygiene so helpful in orthodontic treatment.

P.A., J.B., F.H. What role, in your opinion, can lasers play in the treatment of apthous ulcers. These lesions are particularly disabling when patients are wearing fixed appliances.

S.N. Lasers diminish laser diminish the severity of the inflammatory reaction and thus immediately reduce patients' discomfort after the lesion has been irradiated. And then they participate in a speeding-up of the ulceration's healing.

As for simple ulcerations and bruises caused by irritation from the appliance, lasers are quite effective in accelerating their healing. Of course, for that result to be permanent, the practitioner should be certain to remove the causative mechanical irritant.

P.A., J.B., F.H. Let's get back, if you will, to re-contouring the gingiva with a CO2 laser. Would you proceed 
by stretching or lengthening the gingiva or by thinning it out?

S.N. I would begin with removing excess tissue until I obtained the level I wanted (the eventual limit of the cervical gingiva), by making the tissue taut in order to incise it: this means "a cut with a focalized bundle". Then the practitioner completes the procedure with a $\mathrm{CO}_{2}$ weak in power, two or three Watts, to thin out the gingiva in a sweeping movement, because if the tissue is left too thick it will heal with an unaesthetic thick contour. The laser can serve as well in establishing the thickness of the healed gingiva, which, cut to a proper thinness, will retain the desired proportions.

It is important to mention that the practitioner must preserve the integrity of the dental enamel with the aid of a polished metallic instrument as the gingivoplasty is being carried out.

P.A., J.B., F.H. Many recent articles have discussed the possibility of reducing the pain that accompanies orthodontic treatment with low level laser therapy (LLLT) ${ }^{11}$, or by using a $\mathrm{CO}^{3}$ laser. Can you tell us more about this concept?

S.N. The LLLT laser produces no immediately visible effect on irradiated tissue. However, it releases a quantity of energy whose absorption into tissue will have an anti-inflammatory action. In this way it might reduce orthodontic pain that is inflammatory in origin.

P.A., J.B., F.H. What about the use of a $\mathrm{CO}_{2}$ laser?

S.N. Currently physiotherapists are using the anti-inflammatory action of $\mathrm{CO}_{2}$ lasers to treat lesions and pain that result from inflammation. To obtain this effect, they direct a low level defocalizing $\mathrm{CO}_{2}$ laser beam at the selected site. Dentists can use the same procedure by aiming the defocalizing low level, 0.2 Watts at most, $\mathrm{CO}_{2}$ beam from a distance of $\pm 3 \mathrm{~cm}$ at the treatment site. The irradiation must be delivered with a circular movement around the chosen area rapidly, $\pm 1 \mathrm{~cm} / \mathrm{sec}$, for a period of 1 to 3 minutes. The practitioner can stop irradiating the area as soon as a change in color, from a deep red to lighter shade, is perceived. The same treatment can be repeated every other day, three times a week, if necessary.

P.A., J.B., F.H. The results of a preliminary study, published last year by Youssef, et al. ${ }^{12}$, seem to suggest that the action of a soft laser (LLLT) can help teeth to move faster in response to orthodontic force. What do you think about that?

S.N. Lasers act on tissues by improving the activity of the cells they reach. Thus, it is plausible to imagine, as the osteoclasts and osteoblasts perform more effectively, that the phenomenon of apposition-resorption might accelerate and speed up the rate of tooth movement.

P.A., J.B., F.H. In the treatment of some malocclusions, especially of adults whose periodontium has, not infrequently, begun to deteriorate, orthodontists have to deal with the problem of denudation of root surfaces and the pain that accompanies it. Can you tell us something about the use of the Nd:Yag laser in a pulsating mode for desensitizing exposed cementum?

S.N. Our team has developed a protocol which stipulates choice of appropriate wave lengths adapted to 
fuse the affected dental surface and thus close up entry to dentinal tubules. This superficial tubular blockage, which prevents any flow of liquids, confers desensitization that lasts for at least twelve months, which happens to be the exact length of time our study has been active. So, of course, the relief could be much longer.

P.A., J.B., F.H. So the Nd: Yag is the laser of choice for this type of procedure?

S.N. Precisely. Its photothermal action fuses the dentinal surface by heating the tissues, which takes care of the problem of exposed root surface. But the effect is not just superficial and the pulp could be at risk from deep penetration of heat because the liquid present in the dentine does not absorb $\mathrm{Nd}$ :YAG laser beams to any useful extent. Fortunately we have learned how to limit the diffusion of the laser heat without weakening its effect on the exposed root surface. To do that we cover the root with a black graphite based gel that absorbs the unwanted $\mathrm{Nd}$ : YAG beams without interfering with surface action.

P.A., J.B., F.H. In view of the published studies that indicate adhesion of bonding agents to enamel surfaces etched with the Er:YAG laser is inferior ${ }^{7}$ to or, at best, équivalent ${ }^{5}$ to surfaces etched with acid, do you think that orthodontists ought to use lasers for this purpose?

S.N. Yes, these studies do show that when only the Erbium -YAG laser is used to etch enamel, the results are not optimal. But when it is combined with traditional phosphoric acid etching of enamel, there is a significant increase in bond strength ${ }^{5}$.

However, our team at the University of Liege is now developing an entirely new protocol in which we condition the enamel by irradiation with the Er: YAG laser in a pre-determined fashion. We follow this conditioning with acid etching. The bonding carried out on this surface is superior, by $50 \%$, to that obtained by traditional etching with no preparatory laser conditioning. We have submitted this study to the journal "Lasers in Medical Science" for future publication.

P.A., J.B., F.H. Orthodontists can select ceramic brackets as a more esthetically alternative to metallic attachments in their treatment of malocclusions. Unfortunately their weak resistance to fracture and the strength of their adhesion to enamel, which makes them hard to remove at the termination of therapy, are serious drawbacks. One suggestion to facilitate debonding is to heat them ${ }^{10}$. But poor control of the heat level during this process might put the dental pulp at risk. Do you think $\mathrm{CO}_{2}$ laser can be used safely and effectively in the removal of ceramic brackets?

S.N. The $\mathrm{CO}_{2}$ laser can, in fact, be used for this indication. The orthodontist aims the beam at the bracket, which will absorb the heat and transfer it to the bonding agent causing it to soften and lose its grip on the bracket. You are quite right to bring up the risk to the dental pulp but this can be avoided if the practitioner takes pains to protect the enamel surface with an instrument, for example that would 
absorb excess heat and channel it away from the tooth surface.

P.A., J.B., F.H. Are you confirming that ceramic brackets will effectively absorb the CO2 laser beam?

S.N. Certainly.

P.A., J.B., F.H. White spots left on the enamel surface at the close of treatment can compromise the esthetic result of orthodontic treatment. Knösel, et al. ${ }^{4}$. have suggested that orthodontists can attenuate the unaesthetic impact of white spots by external whitening of the dentition. Do you think orthodontists could use the KTP laser as an effective solution in treating patients who have this problem?

S.N. Definitely. Dentists can whiten teeth very effectively by working with a KTP laser and a gel precisely placed and well adapted. I am proud to be able to recommend our own gel, which is made in Belgium, Smartbleach ${ }^{*}$. It contains rhodamine, whose red coloration absorbs the energy emitted by the KTP and thus prevents over-heating of the dental pulp.

P.A., J.B., F.H. RJ. Lanzafame has brought up the question of the cost to dentists of buying lasers ${ }^{6}$. Do you think the high price of this Investment is justified?

S.N. Dentists can justify an investment of this type if they choose a laser truly adapted to their clinical needs. $\mathrm{A} \mathrm{CO}_{2}$ laser, for example, would be appropriate for orthodontists because it works well for tasks they can be called upon to perform such as re-contouring gingival tissue, frenec-

*Smartbleach International / Mutsaardstraat 47 / 9550. Herzele België. tomies, exposing impacted teeth, and debonding ceramic brackets. But, of course, if you select a type of a laser that does not respond to your practice needs, you are sure to suffer from buyer's remorse.

P.A., J.B., F.H. Do you think the price of lasers will come down in the near future?

S.N. Certainly. Their price will decrease as the demand for them increases. In fact, when supply houses become aware of how many lasers are being sold, more and more of them will want participate in their distribution. This increase in competition combined with the progressive amortization of their cost of development will inevitably lead to a drop in their price.

P.A., J.B., F.H. Dentistry is the last medical specialty to utilize lasers, which are already widely employed in oncology, dermatology, ophthalmology, plastic surgery, and gastroenterology. What are the future prospects for their use the various branches of dentistry?

S.N. Lasers make it possible for dentists to perform certain procedures without great difficulty that were formerly impossible or exquisitely demanding. That is why I am convinced that in time we shall all be working with lasers that have been carefully chosen to fit our precise needs in our daily practice of dentistry.

P.A., J.B., F.H. Thank you very much, Professor Nammour, for the Information you have provided and for the courtesy you have shown to the readers of our Revue.

We are grateful to Fornaini, et al. ${ }^{2}$, the authors of the article in which the photos and case presentations illus- 
trating treatment of impacted teeth and performance of frenectomies appeared.

The photos in figures 2 and 3 are documents that J. Berrebi provided.
Readers will find in the first article in the bibliography an aid to making a reasoned choice in the purchase of a laser that will best fit their needs ${ }^{1}$.

\section{REFERENCES}

1. Berrebi J, Bien choisir son laser un acte raisonné. Rev Orthop Dento Faciale 2009;43:377-83.

2. Fornaini C, Rocca JP, Bertrand MF, Merigo E, Nammour S, Vescovi P. Nd:YAG and Diode Laser in the surgical management of soft tissues related to orthodontic treatment. Photomed Laser Surg 2007;25:381-92.

3. Fujiyama K, Deguchi T, Murakami T, Fujii A, Kushima K, Takano-Yamamoto T.Clinical effect of CO2 laser in reducing pain in orthodontics.Angle Orthod. 2008;78:299-303.

4. Knösel.M, Attin R, Becker K, Attin T. External bleaching effect on the color and luminosity of inactive white-spot lesions after fixed orthodontic appliances. Angle Orthod 2007;77:646-52.

5. Lee BS, Hsieh TT, Lee YL, Lan WH, Hsu YJ, Wen PH, Lin CP.Bond strengths of orthodontic bracket after acid-etched, Er:YAG laser-irradiated and combined treatment on enamel surface.Angle Orthod 2003;73:565-70.

6. Lanzafame RJ. "Business". Photomed Laser Surg. 2007;25:371-2.

7. Martinez-Insua A, Dominguez LDS, Rivera FG, Santana-Penin UA. Differences in bonding to acid- etched or Er:YAG-laser-treated enamel and dentin surfaces. J Prosthet Dent 2000;84:280-8.

8. Nammour S, Demortier G, Florio P, Delhaye Y, Pireaux JJ, Morciaux Y, Powell L. Increase of enamel fluoride retention by low fluence argon laser in vivo. Lasers Surg Med. 2003;33:260-3.

9. Nammour S, Rocca JP, Pireaux JJ, Powell GL, Morciaux Y, Demortier G. Increase of enamel fluoride retention by low fluence argon laser beam: a 6-month follow-up study in vivo. Lasers Surg Med 2005 Mar;36:220-4.

10. Rickabaugh JL, Marangoni RD, McCaffrey KK. Ceramic bracket debonding with the carbon dioxide laser. Am J Orthod Dentofacial Orthop. 1996;110:388-93.

11. Turhani D, Scheriau M, Kapral D, Benesch T, Jonke E, Bantleon HP. Pain relief by single low-level laser irradiation in orthodontic patients undergoing fixed appliance therapy. Am J Orthod Dentofacial Orthop 2006;130:371-7.

12. Youssef M, Ashkar S, Hamade E, Gutknecht N, Lampert F, Mir M. The effect of lowlevel laser therapy during orthodontic movement: a preliminary study. Lasers Med Sci 2008;23:27-33. 